Homology, Homotopy and Applications, vol.12(2), 2010, pp.1-23

\title{
GENERALIZED STEENROD HOMOLOGY THEORIES ARE STRONG SHAPE INVARIANT
}

\author{
PETER MROZIK
}

(communicated by Brooke Shipley)

\begin{abstract}
It is shown that a reduced homology theory on the category of pointed compact metric spaces is strong shape invariant if and only if its homology functors $h_{n}$ satisfy the quotient exactness axiom, which means that for each pointed compact metric pair $\left(X, A, a_{0}\right)$ the natural sequence $h_{n}\left(A, a_{0}\right) \rightarrow h_{n}\left(X, a_{0}\right) \rightarrow$ $h_{n}(X / A, *)$ is exact. As a consequence, all generalized Steenrod homology theories are strong shape invariant.
\end{abstract}

\section{Introduction}

The concept of a generalized Steenrod homology theory for compact metric spaces was introduced in [13] by Kaminker and Schochet. Their purely axiomatic approach distills the essential properties which are common to classical (ordinary) Steenrod homology and to Brown-Douglas-Fillmore homology $\epsilon_{*}$ whose origin lies in the theory of operator algebras (see, e.g., [3]).

Steenrod homology theories are reduced theories defined for single spaces. In [13] the authors dealt with the unpointed case. In the subsequent paper [14], Kahn, Kaminker and Schochet switched to the pointed case which is the more natural setting for reduced homology and on which we shall focus.

By a reduced pointed homology system on the category $\mathbf{C M}_{\mathbf{0}}$ of pointed compact metric spaces we denote any system $\left(h_{n}, \sigma_{n}\right)_{n \in \mathbb{Z}}$ of covariant homotopy invariant functors $h_{n}: \mathbf{C M}_{\mathbf{0}} \rightarrow \mathbf{A b}$ (= category of abelian groups) and of natural isomorphisms $\sigma_{n}: h_{n} \rightarrow h_{n+1} \circ \mathbf{S}$, where $\mathbf{S}$ denotes the reduced suspension functor on $\mathbf{C M}_{\mathbf{0}}$. A reduced pointed homology system is called exact if it satisfies the following exactness axiom: For each pointed compact metric pair $\left(X, A, a_{0}\right)$ the natural sequence

$$
h_{n}\left(A, a_{0}\right) \stackrel{i_{*}}{\longrightarrow} h_{n}\left(X, a_{0}\right) \stackrel{j_{*}}{\longrightarrow} h_{n}\left(X \cup C\left(A, a_{0}\right), *\right)
$$

is exact for all $n \in \mathbb{Z}$.

Here, $\left(X \cup C\left(A, a_{0}\right), *\right)$ denotes the pointed adjunction space obtained by attaching the reduced cone $C\left(A, a_{0}\right)$ to $X$ via the inclusion $A \hookrightarrow C\left(A, a_{0}\right)$.

Received January 17, 2010, revised May 8, 2010; published on July 7, 2010.

2000 Mathematics Subject Classification: 55P55, 55N20, 55N40.

Key words and phrases: Steenrod homology theory, pointed strong shape theory, strong excision axiom, cone collapsing axiom, quotient exactness axiom.

This article is available at http://intlpress.com/HHA/v12/n2/a1

Copyright (c) 2010, International Press. Permission to copy for private use granted. 
In the literature, exact reduced pointed homology systems are customarily denoted as reduced pointed homology theories (see, e.g., [5, 19]), and we shall adopt this notation here.

We now come to a "stronger" variant of exactness. A reduced pointed homology system $\left(h_{n}, \sigma_{n}\right)_{n \in \mathbb{Z}}$ on $\mathbf{C M}_{\mathbf{0}}$ is called quotient exact if it satisfies the following quotient exactness axiom: For each pointed compact metric pair $\left(X, A, a_{0}\right)$ the natural sequence

$$
h_{n}\left(A, a_{0}\right) \stackrel{i_{*}}{\longrightarrow} h_{n}\left(X, a_{0}\right) \stackrel{p_{*}}{\longrightarrow} h_{n}(X / A, *)
$$

is exact for all $n \in \mathbb{Z}$.

Each quotient exact reduced pointed homology system yields a commutative diagram

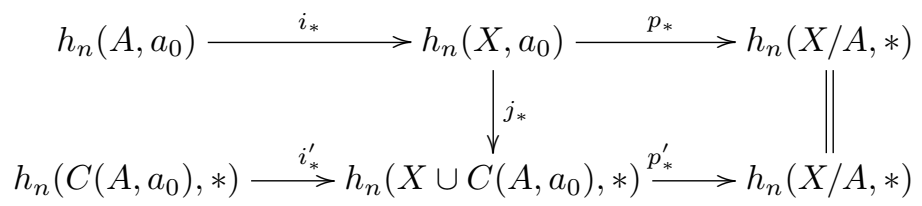

with exact rows. Since $h_{n}\left(C\left(A, a_{0}\right), *\right)=h_{n}(*, *)=0, p_{*}^{\prime}$ is a monomorphism which implies that the exactness axiom is satisfied. That is, each quotient exact reduced pointed homology system is a reduced pointed homology theory. This legitimizes the use of the phrase homology theory in the following definition (cf. [14]).

A Steenrod homology theory on $\mathbf{C M}_{\mathbf{0}}$ is a reduced pointed homology system $\left(h_{n}, \sigma_{n}\right)_{n \in \mathbb{Z}}$ on $\mathbf{C M}_{\mathbf{0}}$, which is quotient exact and, in addition, satisfies the following cluster axiom: For each sequence $\left(X_{i}, x_{i}\right)_{i \in \mathbb{N}}$ of pointed compacta, the projections $p_{k}: \mathbf{C l}_{i=1}^{\infty}\left(X_{i}, x_{i}\right) \rightarrow\left(X_{k}, x_{k}\right)$ defined on the cluster $\mathbf{C l}_{i=1}^{\infty}\left(X_{i}, x_{i}\right)$ induce isomorphisms

$$
\pi_{n}: h_{n}\left(\mathbf{C l}_{i=1}^{\infty}\left(X_{i}, x_{i}\right)\right) \rightarrow \prod_{k=1}^{\infty} h_{n}\left(X_{k}, x_{k}\right)
$$

for all $n \in \mathbb{Z}$.

An abundance of Steenrod homology theories is constructed in [14] by associating to any $\mathrm{CW}$-spectrum $\underline{E}$ a Steenrod homology theory ${ }^{s} \underline{E}_{*}$, which is an extension of the standard reduced pointed homology theory $\underline{E}_{*}$ defined on the category $\mathbf{C W}_{\mathbf{0}}^{\text {fin }}$ of finite pointed CW-complexes (see, e.g., [19, Section 8]). Therefore each reduced pointed homology theory $h_{*}$ on $\mathbf{C W}_{\mathbf{0}}^{\text {fin }}$ admits a Steenrod extension ${ }^{s} h_{*}$ to $\mathbf{C M}_{\mathbf{0}}$ because $h_{*}$ can be represented by a CW-spectrum.

In [6, Section 8.2] Edwards and Hastings give an alternative construction of a Steenrod extension ${ }^{s} h_{*}$ of a reduced pointed homology theory $h_{*}$ on $\mathbf{C W}_{\mathbf{0}}^{\text {fin }}$. Their approach brings together Steenrod extensions and strong shape theory by factorizing ${ }^{s} h_{*}$ over the Vietoris functor $V: \mathbf{C M}_{\mathbf{0}} \rightarrow \mathbf{H o}(\mathbf{p r o}-\mathbf{S S})$. Since $V$ is strong shape invariant, the Steenrod extensions defined in [6] are also strong shape invariant.

For a deeper understanding of Steenrod extensions and their relation to strong shape theory, see Bauer's papers $[\mathbf{1}, \mathbf{2}]$.

It is worth mentioning that ordinary Steenrod homology theory is also known to be strong shape invariant. This is excellently explained in Ferry's paper [7]. 
The object of this paper is to show that all Steenrod homology theories are strong shape invariant. This will be derived directly from the axioms by continuing the approach of [18], where we showed that, among unreduced homology theories on the category of compact metric pairs, the strong shape invariant theories are precisely those satisfying the strong excision axiom. Our main result is the following.

Strong Shape Invariance Theorem (Theorem 3.5): The strong shape invariant reduced pointed homology theories on $\mathbf{C M}_{\mathbf{0}}$ are precisely the quotient exact reduced pointed homology systems. In particular, all Steenrod homology theories on $\mathbf{C M}_{\mathbf{0}}$ are strong shape invariant.

The proof of the Strong Shape Invariance Theorem is worth sketching here because of its exceptional clarity.

The reason why exactness plus strong shape invariance imply quotient exactness is that quotient maps collapsing a contractible subspace to a point are strong shape equivalences. This yields an isomorphism $q_{*}: h_{n}\left(X \cup C\left(A, a_{0}\right), *\right) \rightarrow h_{n}(X / A, *)$, which results in quotient exactness.

The main ingredient in the proof of the converse is a representation of the pointed strong shape category of pointed compacta $\mathbf{S S h}_{\mathbf{0}}$ as a quotient category: In Section 7 we show that the strong shape functor $S: \mathbf{C M}_{\mathbf{0}} \rightarrow \mathbf{S S h}_{\mathbf{0}}$ localizes $\mathbf{C M}_{\mathbf{0}}$ at the class of pointed cylinder base embeddings. A characteristic feature of pointed cylinder base embeddings $i:\left(A, a_{0}\right) \hookrightarrow\left(X, a_{0}\right)$ is that they are inclusions such that the quotient space $(X / A, *)$ is pointed contractible (see Proposition 7.4). But now there is a really remarkable coincidence: Any quotient exact reduced pointed homology system on $\mathbf{C M}_{\mathbf{0}}$ takes such inclusions to isomorphisms which proves its strong shape invariance.

Although in this paper we concentrate on reduced pointed homology theories, we also treat reduced unpointed homology theories and obtain an analogue of the Strong Shape Invariance Theorem (Theorem 4.3). Moreover, in Section 6 we show that:

- strong shape invariant reduced pointed homology theories,

- strong shape invariant reduced unpointed homology theories, and

- strong shape invariant unreduced homology theories

are equivalent which means that they can be transformed into each other without losing information.

In particular, we see that the strong excision axiom in the unreduced case and the quotient exactness axiom in the reduced case are equivalent characterizations of the same phenomenon - namely strong shape invariance - which may possibly lead to a deeper understanding of the general context of these axioms.

It is also interesting that strong shape invariant homology theories occur in the entourage of the Novikov conjecture. To understand why ordinary Steenrod homology theory arises in studies of the Novikov Conjecture, the reader should consult [7]. In the most general form, strong shape invariant homology theories appear in Higson's and Roe's paper [11] on the coarse Baum-Connes conjecture. In [11], the authors consider coarsenings of generalized homology theories $M_{*}$. In Proposition 4.3, which states that the coarsening map $c: M_{*}(\mathcal{O Y}) \rightarrow M X_{*}(\mathcal{O} Y)$ is an isomorphism for any finite-dimensional compact metric space $Y$, they have to assume that the homology theory $M_{*}$ satisfies the strong excision axiom, i.e., is strong shape invariant. 


\section{Reduced homology theories: Pointed case}

Reduced homology theories can be defined for pointed and unpointed spaces. We concentrate on the pointed case since this is the "natural setting" for reduced homology. The unpointed case will be addressed in short form in Section 4.

In the sequel we shall need the four functors:

- Abs: $\mathbf{C M}_{\mathbf{0}}^{\mathbf{2}} \rightarrow \mathbf{C M}_{\mathbf{0}}, \operatorname{Abs}\left(X, A, a_{0}\right)=\left(X, a_{0}\right), \operatorname{Abs}(f)=f$,

- Res: $\mathbf{C M}_{\mathbf{0}}^{\mathbf{2}} \rightarrow \mathbf{C M}_{\mathbf{0}}, \operatorname{Res}\left(X, A, a_{0}\right)=\left(A, a_{0}\right), \operatorname{Res}(f)=\left.f\right|_{A}$,

- $\Gamma: \mathbf{C M}_{\mathbf{0}}^{\mathbf{2}} \rightarrow \mathbf{C M}_{\mathbf{0}}, \Gamma\left(X, A, a_{0}\right)=\left(X \cup C\left(A, a_{0}\right), *\right), \Gamma(f)=f \cup C\left(\left.f\right|_{A}\right)$,

- $Q: \mathbf{C M}_{\mathbf{0}}^{2} \rightarrow \mathbf{C M}_{\mathbf{0}}, Q\left(X, A, a_{0}\right)=(X / A, *), Q(f)=$ induced quotient map.

The above functors come along with four natural transformations:

- Inclusion transformation $i: \operatorname{Res} \rightarrow \operatorname{Abs}, i^{\left(X, A, a_{0}\right)}:\left(A, a_{0}\right) \rightarrow\left(X, a_{0}\right)$,

- Inclusion transformation $j: \operatorname{Abs} \rightarrow \Gamma, j^{\left(X, A, a_{0}\right)}:\left(X, a_{0}\right) \rightarrow\left(X \cup C\left(A, a_{0}\right), *\right)$,

- Quotient transformation $p: \operatorname{Abs} \rightarrow Q, p^{\left(X, A, a_{0}\right)}:\left(X, a_{0}\right) \rightarrow(X / A, *)$,

- Quotient transformation $q: \Gamma \rightarrow Q, q^{\left(X, A, a_{0}\right)}:\left(X \cup C\left(A, a_{0}\right), *\right) \rightarrow(X / A, *)=$ $\left(\left(X \cup C\left(A, a_{0}\right)\right) / C\left(A, a_{0}\right), *\right)$.

In the following definitions let $h: \mathbf{C M}_{\mathbf{0}} \rightarrow \mathbf{A b}$ be an arbitrary covariant functor.

Definition 2.1 (Exactness Axiom). $h$ satisfies the exactness axiom if for all pointed pairs $\left(X, A, a_{0}\right) \in \mathbf{C M}_{\mathbf{0}}^{2}$ the sequence

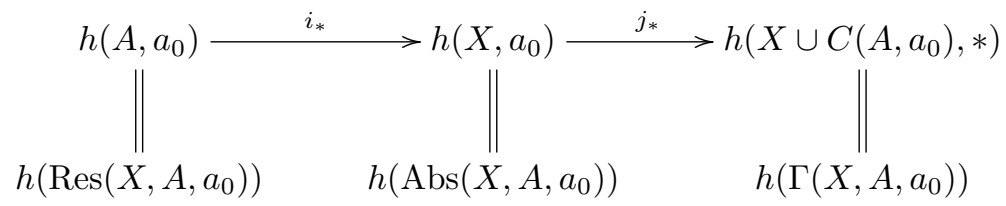

is exact.

Definition 2.2 (Quotient Axiom). $h$ satisfies the quotient exactness axiom if for all pointed pairs $\left(X, A, a_{0}\right) \in \mathbf{C M}_{\mathbf{0}}^{2}$ the sequence

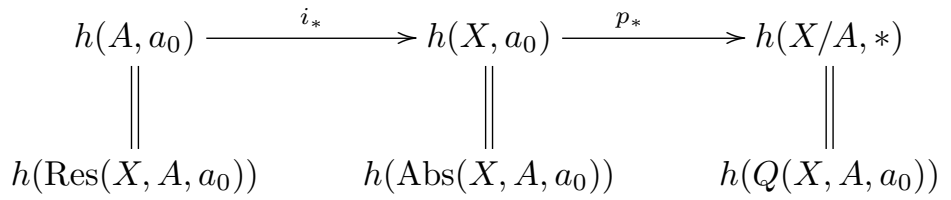

is exact.

Definition 2.3 (Cone Collapsing Axiom). $h$ satisfies the cone collapsing axiom if for all pointed pairs $\left(X, A, a_{0}\right) \in \mathbf{C M}_{\mathbf{0}}^{2}$ the quotient map $q:\left(X \cup C\left(A, a_{0}\right), *\right) \rightarrow(X / A, *)$ induces an isomorphism

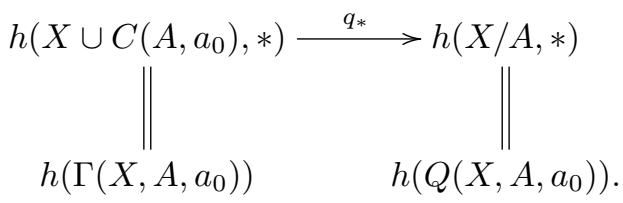


The following result (which is essentially due to [3]) relates the above concepts.

Theorem 2.4. Let $h$ be homotopy invariant. Then the following are equivalent:

(1) $h$ satisfies the quotient exactness axiom.

(2) $h$ satisfies the cone collapsing axiom and the exactness axiom.

Proof of $(1) \Rightarrow(2)$. The cone collapsing axiom follows from the proofs of $[\mathbf{3}$, Lemmas 2.16 and 2.17]. These proofs deal with $h=$ Ext in the unpointed case. The only properties of Ext used in [3] are homotopy invariance and quotient exactness so that the proofs apply to our situation. Replacing unreduced mapping cylinders, mapping cones, cones and suspensions by their reduced versions, we see that if $\left(X, A, a_{0}\right) \in \mathbf{C M}_{\mathbf{0}}^{2}$ is a pointed pair such that $\left(A, a_{0}\right)$ is pointed contractible, then $p_{*}: h\left(X, a_{0}\right) \rightarrow h(X / A, *)$ is an isomorphism. The cone collapsing axiom is just a special case of this result.

The exactness axiom is satisfied because $p_{*}=q_{*} \circ j_{*}$.

Proof of $(2) \Rightarrow(1)$. This is obvious since $p_{*}=q_{*} \circ j_{*}$.

\section{Definition 2.5.}

1. A reduced pointed homology system on $\mathbf{C M}_{\mathbf{0}}$ is a collection of homotopy invariant covariant functors $h_{n}: \mathbf{C M}_{\mathbf{0}} \rightarrow \mathbf{A b}$ and natural isomorphisms $\sigma_{n}: h_{n} \rightarrow$ $h_{n+1} \circ \mathbf{S}, n \in \mathbb{Z}$.

2. A reduced pointed homology theory is a reduced pointed homology system such that all homology functors $h_{n}$ satisfy the exactness axiom.

3. A quotient exact reduced pointed homology system is a reduced pointed homology system such that all homology functors $h_{n}$ satisfy the quotient exactness axiom.

From Theorem 2.4 we derive

Corollary 2.6. On $\mathbf{C M}_{\mathbf{0}}$ the quotient exact reduced pointed homology systems coincide with the reduced pointed homology theories which satisfy the cone collapsing axiom (i.e., for which all homology functors $h_{n}$ satisfy the cone collapsing axiom).

One can give an alternative definition of a reduced pointed homology theory as a collection of homotopy invariant covariant functors $h_{n}: \mathbf{C M}_{\mathbf{0}} \rightarrow \mathbf{A b}$ and natural transformations $\partial_{n}: h_{n+1} \circ \Gamma \rightarrow h_{n} \circ$ Res satisfying the long exactness axiom, which means that for each $\left(X, A, a_{0}\right) \in \mathbf{C M}_{\mathbf{0}}^{\mathbf{2}}$ there is a long exact sequence

$$
\begin{aligned}
\cdots \stackrel{\partial_{n}}{\longrightarrow} h_{n}\left(A, a_{0}\right) \stackrel{i_{*}}{\longrightarrow} h_{n}\left(X, a_{0}\right) \stackrel{j_{*}}{\longrightarrow} h_{n}\left(X \cup C\left(A, a_{0}\right), *\right) \\
\stackrel{\partial_{n-1}}{\longrightarrow} h_{n-1}\left(A, a_{0}\right) \stackrel{i_{*}}{\longrightarrow} \cdots .
\end{aligned}
$$

To verify the equivalence of both definitions we need the natural transformation

$$
\xi: \Gamma \rightarrow \mathbf{S} \circ \operatorname{Res}
$$

given by the maps

$$
\begin{aligned}
\xi^{\left(X, A, a_{0}\right)}:\left(X \cup C\left(A, a_{0}\right), *\right) \stackrel{\pi}{\longrightarrow}\left(\left(X \cup C\left(A, a_{0}\right)\right) / X, *\right) & \\
& =\left(C\left(A, a_{0}\right) / A, *\right) \stackrel{h}{\rightarrow} \mathbf{S}\left(A, a_{0}\right),
\end{aligned}
$$

where $\pi$ is the quotient map and $h$ is the obvious homeomorphism. Note that the 
inclusion $X \hookrightarrow X \cup C\left(A, a_{0}\right)$ is a cofibration. Hence $\xi^{\left(X, A, a_{0}\right)}$ is a pointed homotopy equivalence if $\left(X, a_{0}\right)$ is pointed contractible.

Given a reduced pointed homology theory in the sense of the original definition, we define $\partial_{n}=\partial\left(\sigma_{n}\right)$ as the composition

$$
h_{n+1}\left(X \cup C\left(A, a_{0}\right), *\right) \stackrel{\xi_{*}}{\longrightarrow} h_{n+1}\left(\mathbf{S}\left(A, a_{0}\right)\right) \stackrel{\sigma_{n}^{-1}}{\longrightarrow} h_{n}\left(A, a_{0}\right) .
$$

The proof of long exactness can be found in [19, Section 7.33]. Observe that [19] gives a different definition of $\partial\left(\sigma_{n}\right)$ by interposing the automorphism $\nu_{*}: h_{n+1}\left(\mathbf{S}\left(A, a_{0}\right)\right) \rightarrow$ $h_{n+1}\left(\mathbf{S}\left(A, a_{0}\right)\right)$ induced by the homotopy inverse $\nu$ on the $h$-cogroup $\mathbf{S}\left(A, a_{0}\right)$. That is, $\partial\left(\sigma_{n}\right)$ as defined here is nothing else but $\partial\left(\nu_{*} \circ \sigma_{n}\right)$ as defined in $[\mathbf{1 9}]$. But $\left(h_{n}, \nu_{*} \circ \sigma_{n}\right)$ is also a reduced pointed homology theory so we get the desired exactness result.

Conversely, given a reduced pointed homology theory in the alternative sense, we can construct natural isomorphisms $\sigma_{n}=\sigma\left(\partial_{n}\right)$ as follows: The long exact sequence of the pointed pair $\left(C\left(A, a_{0}\right), A, a_{0}\right)$ yields isomorphisms

$$
h_{n+1}\left(\Gamma\left(C\left(A, a_{0}\right), A, a_{0}\right) \stackrel{\partial_{n}}{\longrightarrow} h_{n}\left(A, a_{0}\right)\right.
$$

since all $h_{n}\left(C\left(A, a_{0}\right), *\right)=0$. We can therefore define

$$
\sigma_{n}: h_{n}\left(A, a_{0}\right) \stackrel{\partial_{n}^{-1}}{\longrightarrow} h_{n+1}\left(\Gamma\left(C\left(A, a_{0}\right), A, a_{0}\right)\right) \stackrel{\xi_{*}}{\longrightarrow} h_{n+1}\left(\mathbf{S}\left(A, a_{0}\right)\right) .
$$

In the above situation $\xi$ is a pointed homotopy equivalence because $C\left(A, a_{0}\right)$ is pointed contractible. Therefore $\sigma_{n}$ is a natural isomorphism.

The assignments $\sigma_{n} \mapsto \partial\left(\sigma_{n}\right)$ and $\partial_{n} \mapsto \sigma\left(\partial_{n}\right)$ are inverse to each other; i.e., we have $\sigma\left(\partial\left(\sigma_{n}\right)\right)=\sigma_{n}$ and $\partial\left(\sigma\left(\partial_{n}\right)\right)=\partial_{n}$.

To verify the first equation observe that $\xi_{*}=\xi_{*}^{\left(C\left(A, a_{0}\right), A, a_{0}\right)}$ is an isomorphism whence

$$
\begin{aligned}
\sigma\left(\partial\left(\sigma_{n}\right)\right) & =\xi_{*}^{\left(C\left(A, a_{0}\right), A, a_{0}\right)} \circ \partial\left(\sigma_{n}\right)^{-1} \\
& =\xi_{*}^{\left(C\left(A, a_{0}\right), A, a_{0}\right)} \circ\left(\left(\sigma_{n}\right)^{-1} \circ \xi_{*}^{\left(C\left(A, a_{0}\right), A, a_{0}\right)}\right)^{-1} \\
& =\sigma_{n} .
\end{aligned}
$$

To check the second equation, let us consider the inclusion maps $\mu:\left(X, A, a_{0}\right) \rightarrow$ $\left(C\left(X, a_{0}\right), A, a_{0}\right)$ and $\nu:\left(C\left(A, a_{0}\right), A, a_{0}\right) \rightarrow\left(C\left(X, a_{0}\right), A, a_{0}\right)$. The following diagram commutes because $\xi$ and $\partial_{n}$ are natural transformations:

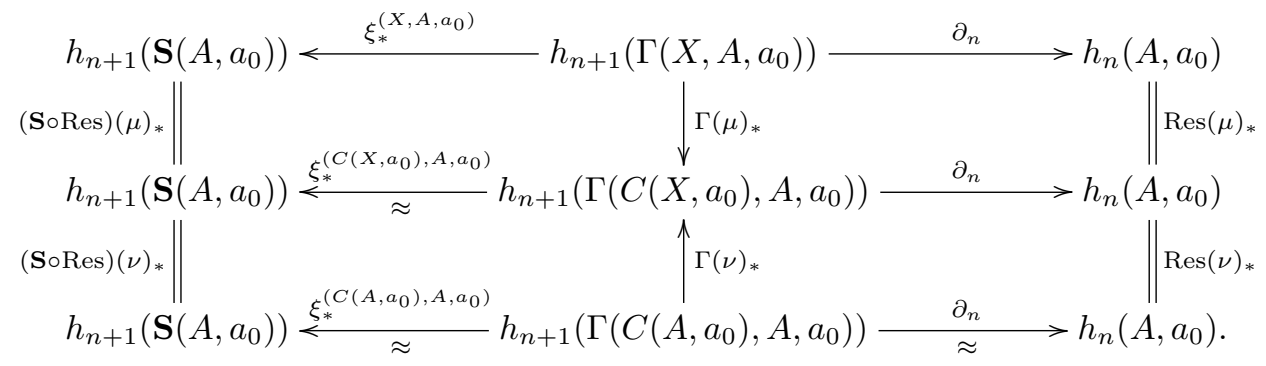


This implies

$$
\begin{aligned}
\partial\left(\sigma\left(\partial_{n}\right)\right) & =\sigma\left(\partial_{n}\right)^{-1} \circ \xi_{*}^{\left(X, A, a_{0}\right)}=\left(\xi_{*}^{\left(C\left(A, a_{0}\right), A, a_{0}\right)} \circ \partial_{n}^{-1}\right)^{-1} \circ \xi_{*}^{\left(X, A, a_{0}\right)} \\
& =\partial_{n} \circ\left(\xi_{*}^{\left(C\left(A, a_{0}\right), A, a_{0}\right)}\right)^{-1} \circ \xi_{*}^{\left(X, A, a_{0}\right)} \\
& =\partial_{n} \circ \Gamma(\nu)_{*} \circ\left(\xi_{*}^{\left(C\left(A, a_{0}\right), A, a_{0}\right)}\right)^{-1} \circ \xi_{*}^{\left(X, A, a_{0}\right)} \\
& =\partial_{n} \circ\left(\xi_{*}^{\left(C\left(X, a_{0}\right), A, a_{0}\right)}\right)^{-1} \circ \xi_{*}^{\left(X, A, a_{0}\right)} \\
& =\partial_{n} \circ \Gamma(\mu)_{*}=\partial_{n} .
\end{aligned}
$$

Let us finally consider yet another concept of exactness.

Definition 2.7. A reduced pointed Q-homology theory on $\mathbf{C M}_{\mathbf{0}}$ is a collection of homotopy invariant covariant functors $h_{n}: \mathbf{C M}_{\mathbf{0}} \rightarrow \mathbf{A b}$ and natural transformations $\Delta_{n}: h_{n+1} \circ Q \rightarrow h_{n} \circ$ Res, $n \in \mathbb{Z}$, such that for each $\left(X, A, a_{0}\right) \in \mathbf{C M}_{\mathbf{0}}^{2}$ there is a long exact sequence

$$
\cdots \stackrel{\Delta_{n}}{\longrightarrow} h_{n}\left(A, a_{0}\right) \stackrel{i_{*}}{\longrightarrow} h_{n}\left(X, a_{0}\right) \stackrel{p_{*}}{\longrightarrow} h_{n}(X / A, *) \stackrel{\Delta_{n-1}}{\longrightarrow} h_{n-1}\left(A, a_{0}\right) \stackrel{i_{*}}{\longrightarrow} \cdots .
$$

Lemma 2.8. Each reduced pointed Q-homology theory $\left(h_{n}, \Delta_{n}\right)$ satisfies the cone collapsing axiom (i.e., all functors $h_{n}$ satisfy the cone collapsing axiom).

Proof. The long exact sequence of the pointed pair $\left(X \cup C\left(A, a_{0}\right), C\left(A, a_{0}\right), *\right)$ shows that all

$$
q_{*}: h_{n}\left(X \cup C\left(A, a_{0}\right), *\right) \rightarrow h_{n}(X / A, *)
$$

are isomorphisms.

Our above alternative definition of a reduced pointed homology theory immediately yields the following corollary which is closely related to [3, Theorem 2.19].

Corollary 2.9. On $\mathbf{C M}_{\mathbf{0}}$ the reduced pointed Q-homology theories $\left(h_{n}, \Delta_{n}\right)$ can be identified with the reduced pointed homology theories $\left(h_{n}, \partial_{n}\right)$ which satisfy the cone collapsing axiom. The identification is given by the assignment

$$
\Delta_{n} \mapsto \partial_{n}=\Delta_{n} \circ q_{*} .
$$

\section{Reduced pointed homology theories on $\mathrm{CM}_{0}$ and strong shape invariance}

Definition 3.1 (Strong Shape Invariance Axiom). A reduced pointed homology system $\left(h_{n}, \sigma_{n}\right)$ on $\mathbf{C M}_{\mathbf{0}}$ is strong shape invariant if each pointed strong shape equivalence $f:\left(X, x_{0}\right) \rightarrow\left(Y, y_{0}\right)$ between pointed compacta induces isomorphisms

$$
f_{*}: h_{n}\left(X, x_{0}\right) \rightarrow h_{n}\left(Y, y_{0}\right)
$$

for all $n \in \mathbb{Z}$.

Remark 3.2. The strong shape functor $S: \mathbf{C M}_{\mathbf{0}} \rightarrow \mathbf{S S h}_{\mathbf{0}}$ localizes $\mathbf{C M}_{\mathbf{0}}$ at the class of pointed strong shape equivalences (see Section 7). As a consequence, for each reduced pointed homology system $\left(h_{n}, \sigma_{n}\right)$ on $\mathbf{C M}_{\mathbf{0}}$, strong shape invariance is equivalent to the existence of covariant functors $h_{n}^{\prime}: \mathbf{S S h}_{\mathbf{0}} \rightarrow \mathbf{A b}$ such that $h_{n}=h_{n}^{\prime} \circ S$ for all $n \in \mathbb{Z}$. These functors $h_{n}^{\prime}$ are of course uniquely determined. 
Theorem 3.3. Let $\left(h_{n}, \sigma_{n}\right)$ be a reduced pointed homology theory on $\mathbf{C M}_{\mathbf{0}}$. Then the following are equivalent:

(1) $\left(h_{n}, \sigma_{n}\right)$ is strong shape invariant.

(2) $\left(h_{n}, \sigma_{n}\right)$ satisfies the cone collapsing axiom.

Proof of $(1) \Rightarrow(2)$. The quotient map $q:\left(X \cup C\left(A, a_{0}\right), *\right) \rightarrow(X / A, *)$ is a pointed strong shape equivalence because $C\left(A, a_{0}\right)$ is contractible see, e.g., $[\mathbf{9}$, Theorem 2 and Corollary 3]).

Proof of $(2) \Rightarrow(1)$. Let $i:\left(A, a_{0}\right) \rightarrow\left(X, x_{0}\right)$ be a pointed cylinder base embedding (cf. Section 7). We shall show that $i$ induces isomorphisms $i_{*}: h_{n}\left(A, a_{0}\right) \rightarrow h_{n}\left(X, x_{0}\right)$ for all $n \in \mathbb{Z}$.

It is no restriction to assume that $A \subset X$ and $a_{0}=x_{0}$ (otherwise decompose $i$ into a homeomorphism and the genuine inclusion from $i\left(A, a_{0}\right)$ into $\left(X, x_{0}\right)$ which is again a pointed cylinder base embedding). Consider the long exact sequence of the pointed pair $\left(X, A, a_{0}\right)$

$$
\begin{aligned}
\cdots \rightarrow h_{n+1}\left(X \cup C\left(A, a_{0}\right), *\right) \stackrel{\partial_{n}}{\longrightarrow} h_{n}\left(A, a_{0}\right) \stackrel{i_{*}}{\rightarrow} h_{n}\left(X, a_{0}\right) & \stackrel{j_{*}}{\longrightarrow} h_{n}\left(X \cup C\left(A, a_{0}\right), *\right) \rightarrow \cdots .
\end{aligned}
$$

By assumption, all $q_{*}: h_{k}\left(X \cup C\left(A, a_{0}\right), *\right) \rightarrow h_{k}(X / A, *)$ are isomorphisms. Since $(X / A, *)$ is pointed contractible (cf. Proposition 7.4), $h_{k}(X / A, *)=0$.

Thus all $h_{k}\left(X \cup C\left(A, a_{0}\right), *\right)=0$, which implies that $i_{*}$ is an isomorphism for all $n \in \mathbb{Z}$.

We have now shown that the $h_{n}$ take pointed cylinder base embeddings to isomorphisms. By Theorem 7.7 there exist $h_{n}^{\prime}: \mathbf{S S h}_{\mathbf{0}} \rightarrow \mathbf{A b}$ such that $h_{n}=h_{n}^{\prime} \circ S$. This implies that the $h_{n}$ take pointed strong shape equivalences to isomorphisms.

Remark 3.4. The implication (1) $\Rightarrow(2)$ holds true for any reduced pointed homology system $\left(h_{n}, \sigma_{n}\right)$. However, the exactness axiom is essential for the proof of $(2) \Rightarrow(1)$, which means that this implication holds true only when $\left(h_{n}, \sigma_{n}\right)$ is a reduced pointed homology theory. Moreover, in the proof $(2) \Rightarrow(1)$ it would be sufficient to assume that the $q_{*}: h_{k}\left(X \cup C\left(A, a_{0}\right), *\right) \rightarrow h_{k}(X / A, *)$ are monomorphisms.

Theorem 3.5. Let $\left(h_{n}, \sigma_{n}\right)$ be a reduced pointed homology system on $\mathbf{C M}_{\mathbf{0}}$. Then the following are equivalent:

(1) $\left(h_{n}, \sigma_{n}\right)$ is quotient exact.

(2) $\left(h_{n}, \sigma_{n}\right)$ is exact and strong shape invariant, i.e., is a strong shape invariant reduced pointed homology theory.

In other words: On $\mathbf{C M}_{\mathbf{0}}$ the quotient exact reduced pointed homology systems coincide with the strong shape invariant reduced pointed homology theories.

Proof of $(1) \Rightarrow(2)$ By Theorem 2.4, $\left(h_{n}, \sigma_{n}\right)$ is exact and satisfies the cone collapsing axiom. Theorem 3.3 then yields strong shape invariance.

Proof. Proof of $(2) \Rightarrow(1)$ By Theorem 3.3, $\left(h_{n}, \sigma_{n}\right)$ satisfies the cone collapsing axiom. Theorem 2.4 yields quotient exactness. 
Corollary 3.6. Each Steenrod homology theory on $\mathbf{C M}_{\mathbf{0}}$ is a strong shape invariant reduced pointed homology theory.

Corollary 3.7. On $\mathbf{C M}_{\mathbf{0}}$ the reduced pointed Q-homology theories $\left(h_{n}, \Delta_{n}\right)$ can be identified with the strong shape invariant reduced pointed homology theories $\left(h_{n}, \partial_{n}\right)$. The identification is given by the assignment

$$
\Delta_{n} \mapsto \partial_{n}=\Delta_{n} \circ q_{*} .
$$

\section{Reduced homology theories: unpointed case}

Reduced theories can also be defined in the unpointed setting. We shall be short since everything is almost identical with the pointed case. In the unpointed case the categories $\mathbf{C M}_{\mathbf{0}}$ (resp. $\mathbf{C M}_{\mathbf{0}}^{\mathbf{2}}$ ) have to be replaced by their unpointed counterparts $\mathbf{C M}_{\text {ne }}$ (resp. $\mathbf{C M}_{\mathbf{n e}}^{\mathbf{2}}$ ) whose objects are all nonempty compact metric spaces $X$ (resp. all compact metric pairs $(X, A)$ such that $A \neq \emptyset)$.

The definitions of the Exactness Axiom, the Quotient Exactness Axiom, the Cone Collapsing Axiom and the Strong Shape Invariance Axiom in the unpointed setting are omitted here since they are completely analogous as in the pointed case (of course, the reduced cone appearing in the Exactness Axiom has to be replaced by the unreduced cone).

\section{Definition 4.1.}

1. A reduced unpointed homology system on $\mathbf{C M}_{\mathbf{n e}}$ is a collection of homotopy invariant covariant functors $h_{n}: \mathbf{C M}_{\mathbf{n e}} \rightarrow \mathbf{A b}$ and natural isomorphisms

$$
\sigma_{n}: h_{n} \rightarrow h_{n+1} \circ \mathbf{S}, n \in \mathbb{Z}
$$

(where $\mathbf{S}: \mathbf{C M}_{\mathbf{n e}} \rightarrow \mathbf{C M}_{\text {ne }}$ is the unreduced suspension functor).

2. A reduced unpointed homology theory is a reduced unpointed homology system such that all homology functors $h_{n}$ satisfy the exactness axiom.

3. A quotient exact reduced unpointed homology system is a reduced unpointed homology system such that all homology functors $h_{n}$ satisfy the quotient exactness axiom.

Our definition of a reduced unpointed homology theory is essentially that given in $[\mathbf{1 2}, \mathbf{1 5}]$ and can therefore be regarded as the "standard" definition.

As in the pointed case there is yet another concept of exactness (see, e.g., [10] and [13, Proposition 2.5]).

Definition 4.2. A reduced unpointed Q-homology theory on $\mathbf{C M}_{\mathbf{n e}}$ is a collection of homotopy invariant covariant functors $h_{n}: \mathbf{C M}_{\mathbf{n e}} \rightarrow \mathbf{A b}$ and natural transformations $\Delta_{n}: h_{n+1} \circ Q \rightarrow h_{n} \circ$ Res, $n \in \mathbb{Z}$, satisfying the axiom of Q-exactness, which says that for each $(X, A) \in \mathbf{C M}_{\mathbf{n e}}^{\mathbf{2}}$ there is a long exact sequence

$$
\cdots \stackrel{\Delta_{n}}{\longrightarrow} h_{n}(A) \stackrel{i_{*}}{\longrightarrow} h_{n}(X) \stackrel{p_{*}}{\longrightarrow} h_{n}(X / A) \stackrel{\Delta_{n-1}}{\longrightarrow} h_{n-1}(A) \stackrel{i_{*}}{\longrightarrow} \cdots .
$$

Let us now state the main results. The proofs are omitted because they can be copied from the pointed case (using [17] instead of Section 7). 
Theorem 4.3. On $\mathbf{C M}_{\mathbf{n e}}$ the following coincide:

- Quotient exact reduced unpointed homology systems,

- Reduced unpointed homology theories which satisfy the cone collapsing axiom,

- Strong shape invariant reduced unpointed homology theories.

The concept of a Steenrod homology theory was originally introduced in $[\mathbf{1 3}]$ for the unpointed case, that is on $\mathbf{C M}_{\mathbf{n e}}$ (see also [6]).

Theorem 4.4. Each Steenrod homology theory on $\mathbf{C M}_{\mathbf{n e}}$ is a strong shape invariant reduced unpointed homology theory.

Theorem 4.5. On $\mathbf{C M}_{\mathbf{n e}}$ the reduced unpointed Q-homology theories $\left(h_{n}, \Delta_{n}\right)$ can be identified with the strong shape invariant reduced unpointed homology theories $\left(h_{n}, \partial_{n}\right)$. The identification is given by the assignment

$$
\Delta_{n} \mapsto \partial_{n}=\Delta_{n} \circ q_{*} .
$$

\section{Strong shape invariance for unreduced homology theories on $\mathrm{CM}^{2}$ revisited}

In [18] we have shown that strong shape invariance and strong excision are equivalent for unreduced homology theories on $\mathbf{C M}^{\mathbf{2}}$. We shall slightly improve this result. In the following definitions, let $\left(H_{n}, \partial_{n}\right)$ be an unreduced homology theory on $\mathbf{C M}^{\mathbf{2}}$.

Definition 5.1 (Cone Collapsing Axiom). $\left(H_{n}, \partial_{n}\right)$ satisfies the cone collapsing axiom if for all $(X, A) \in \mathbf{C M}^{2}$ the quotient map $p:(X \cup C A, C A) \rightarrow(X / A, *)$ induces isomorphisms

$$
p_{*}: H_{n}(X \cup C A, C A) \rightarrow H_{n}(X / A, *)
$$

for all $n \in \mathbb{Z}$.

Remark 5.2. $\left(H_{n}, \partial_{n}\right)$ satisfies the cone collapsing axiom if and only if it satisfies the cone collapsing axiom in the absolute case, which means that $p: X \cup C A \rightarrow X / A$ induces isomorphisms

$$
p_{*}: H_{n}(X \cup C A) \rightarrow H_{n}(X / A)
$$

for all $n \in \mathbb{Z}$.

Proof. Let $(X, A) \in \mathbf{C M}^{2}$. Consider the infinite commutative diagram consisting of the long exact sequences of the pairs $(X \cup C A, C A)$ and $(X / A, *)$ and the homomorphisms $p_{*}$ connecting both sequences. Since all $p_{*}: H_{n}(C A) \rightarrow H_{n}(*)$ are isomorphisms, the five lemma yields the assertion.

Definition 5.3 (Strong Excision Axiom). $\left(H_{n}, \partial_{n}\right)$ satisfies the strong excision axiom if, for all $(X, A) \in \mathbf{C M}^{2}$, the quotient map $p:(X, A) \rightarrow(X / A, *)$ induces isomorphisms

$$
p_{*}: H_{n}(X, A) \rightarrow H_{n}(X / A, *)
$$

for all $n \in \mathbb{Z}$. 
Remark 5.4. In case $A=\emptyset$, we define $(X / \emptyset, *)=\left(X^{+},+\right)$and let

$$
p:(X, \emptyset) \rightarrow(X / \emptyset, *)
$$

denote inclusion. Then $p$ is an excision map (excising the one-point subspace + ) and therefore induces isomorphisms $p_{*}$ in homology.

Definition 5.5. An unreduced homology theory $\left(H_{n}, \partial_{n}\right)$ on $\mathbf{C M}^{\mathbf{2}}$ is strong shape invariant in the absolute case if each strong shape equivalence $f: X \rightarrow Y$ between compacta induces isomorphisms

$$
f_{*}: H_{n}(X) \rightarrow H_{n}(Y)
$$

for all $n \in \mathbb{Z}$.

Theorem 5.6. Let $\left(H_{n}, \partial_{n}\right)$ be an unreduced homology theory on $\mathbf{C M}^{2}$. Then the following are equivalent:

(1) $\left(H_{n}, \partial_{n}\right)$ is strong shape invariant (cf. [18]).

(2) $\left(H_{n}, \partial_{n}\right)$ is strong shape invariant in the absolute case.

(3) $\left(H_{n}, \partial_{n}\right)$ satisfies the cone collapsing axiom.

(4) $\left(H_{n}, \partial_{n}\right)$ satisfies the strong excision axiom.

Proof of $(1) \Rightarrow(2)$. This is obvious.

Proof of $(2) \Rightarrow(3)$. This follows from Remark 5.2 and the fact that $p: X \cup C A \rightarrow X / A$ is a strong shape equivalence (cf. [9]).

Proof of $(3) \Rightarrow(4)$. This follows from the ordinary excision axiom as shown in the proof of [18, Theorem 2.3].

Proof of $(4) \Rightarrow(1)$. This is covered by [18, Theorem 2.3].

\section{The equivalence of unreduced and reduced strong shape invariant homology theories}

It is commonly understood that unreduced and reduced homology theories are equivalent. Unfortunately, it is not true in this general form. In fact, one has to make additional assumptions to establish such an equivalence.

Concerning the equivalence of unreduced and reduced pointed homology theories (which constitute the "natural" framework for reduced homology), the reader should consult [19, Section 7]. The price paid in [19] is to require that homology theories satisfy the weak homotopy axiom and that all pointed spaces under consideration have a non-degenerate basepoint.

The situation is better for reduced unpointed homology theories. Kelly [15] has shown that unreduced homology theories which satisfy the dimension axiom and a slightly weakened form of the excision axiom are equivalent to reduced unpointed homology theories which satisfy a variant of the dimension axiom and a slightly modified form of the exactness axiom (in which $X \cup C A$ is replaced by the space $X \cup A p$ got from $X$ by "joining the subset $A$ to a point $p$ "). For compact metric 
spaces Kelly's modified axioms agree with the standard axioms and it is, moreover, easy to verify that the dimension axiom can be omitted without affecting the proof of the equivalence of unreduced and reduced theories. In the compact metric case we may therefore restate Kelly's results as follows.

Theorem 6.1. Unreduced homology theories on $\mathbf{C M}^{2}$ are equivalent to reduced unpointed homology theories on $\mathbf{C M}_{\mathbf{n e}}$.

For an unreduced theory $\left(H_{n}, \partial_{n}\right)$, the associated reduced theory $\left(\bar{H}_{n}, \sigma\left(\partial_{n}\right)\right)$ is given by

$$
\begin{gathered}
\bar{H}_{n}(X)=H_{n+1}(C X, X) \approx \operatorname{ker}\left(c_{*}: H_{n}(X, \emptyset) \rightarrow H_{n}(*, \emptyset)\right), \\
\bar{H}_{n}(f)=H_{n}(C f) .
\end{gathered}
$$

For a reduced unpointed theory $\left(h_{n}, \sigma_{n}\right)$, the associated unreduced theory $\left(\hat{h}_{n}, \partial\left(\sigma_{n}\right)\right)$ is given by

$$
\begin{aligned}
\hat{h}_{n}(X, A) & =h_{n}(\Gamma(X, A)), \\
\hat{h}_{n}(f) & =h_{n}(\Gamma(f)) .
\end{aligned}
$$

The formulae for $\sigma\left(\partial_{n}\right)$ and $\partial\left(\sigma_{n}\right)$ have been omitted because they are irrelevant for our purposes. Note that in case $A=\emptyset$ we set $\Gamma(X, \emptyset)=X^{+}$.

The purpose of this section is to show that in the realm of compact metric spaces the concepts of

- strong shape invariant unreduced homology theories,

- strong shape invariant reduced unpointed homology theories and

- strong shape invariant reduced pointed homology theories

are completely equivalent.

The first part is obtained as an easy deduction of Theorem 6.1.

Theorem 6.2. Under the equivalence of Theorem 6.1, strong shape invariant unreduced homology theories on $\mathbf{C M}^{\mathbf{2}}$ correspond to strong shape invariant reduced unpointed homology theories on $\mathbf{C M}_{\mathbf{n e}}$.

In other words: Unreduced homology theories on $\mathbf{C M}^{\mathbf{2}}$ which satisfy the strong excision axiom correspond to quotient exact reduced unpointed homology theories on $\mathrm{CM}_{\text {ne. }}$.

Proof. Assume that $\left(H_{n}, \partial_{n}\right)$ is strong shape invariant. Consider a strong shape equivalence $f: X \rightarrow Y$ between nonempty compacta. Then $C(f):(C X, X) \rightarrow(C Y, Y)$ is a strong shape equivalence of pairs; thus $\bar{H}_{n}(f)$ is an isomorphism. This shows that $\left(\bar{H}_{n}, \sigma\left(\partial_{n}\right)\right)$ is strong shape invariant.

Assume that $\left(h_{n}, \sigma_{n}\right)$ is strong shape invariant. Consider a strong shape equivalence $f: X \rightarrow Y$ between compacta. Then also $\Gamma(f)=f^{+}: X^{+} \rightarrow Y^{+}$is a strong shape equivalence; hence $\hat{h}_{n}(f)$ is an isomorphism. This means that $\left(\hat{h}_{n}, \partial\left(\sigma_{n}\right)\right)$ is strong shape invariant in the absolute case and therefore strong shape invariant.

In the reduced pointed case we cannot establish an analogue of Theorem 6.1 but confine ourselves to 
Theorem 6.3. Strong shape invariant unreduced homology theories on $\mathbf{C M}^{\mathbf{2}}$ are equivalent to strong shape invariant reduced pointed homology theories on $\mathbf{C M}_{\mathbf{0}}$.

In other words: Unreduced homology theories on $\mathbf{C M}^{2}$, which satisfy the strong excision axiom, are equivalent to quotient exact reduced pointed homology theories on $\mathrm{CM}_{0}$.

Proof. We establish an equivalence between unreduced homology theories on $\mathbf{C M}^{\mathbf{2}}$ which satisfy the strong excision axiom and reduced pointed Q-homology theories on $\mathbf{C M}_{\mathbf{0}}$. By Corollary 3.7 this proves the theorem.

Given an unreduced homology theory $\left(H_{n}, \partial_{n}\right)$ on $\mathbf{C M}^{2}$, which satisfies the strong excision axiom, we define reduced homology functors $\bar{H}_{n}: \mathbf{C M}_{\mathbf{0}} \rightarrow \mathbf{A b}$ by

$$
\begin{aligned}
\bar{H}_{n}\left(X, x_{0}\right) & =H_{n}\left(X,\left\{x_{0}\right\}\right), \\
\bar{H}_{n}(f) & =H_{n}(f) .
\end{aligned}
$$

For any pointed pair $\left(X, A, a_{0}\right) \in \mathbf{C M}_{\mathbf{0}}^{\mathbf{2}}$ we have a commutative diagram

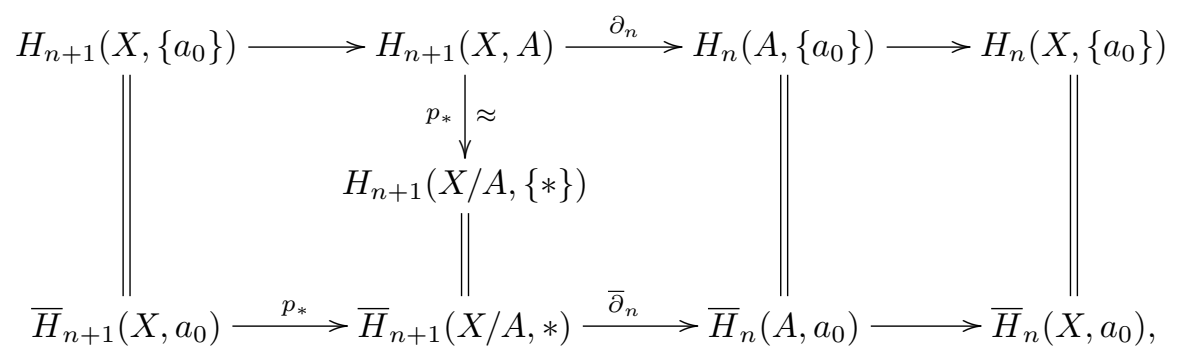

where the top row is the long exact sequence of the triple $\left(X, A,\left\{a_{0}\right\}\right)$, the vertical arrow $p_{*}$ is the strong excision isomorphism and $\bar{\partial}_{n}$ is defined to make the middle square commutative.

It is then clear that $\left(\bar{H}_{n}, \bar{\partial}_{n}\right)$ is a reduced pointed Q-homology theory on $\mathbf{C M}_{\mathbf{0}}$.

Given a reduced pointed Q-homology theory $\left(h_{n}, \Delta_{n}\right)$ on $\mathbf{C M}_{\mathbf{0}}$, we define unreduced homology functors $\hat{h}_{n}: \mathbf{C M}^{2} \rightarrow \mathbf{A b}$ by

$$
\begin{aligned}
\hat{h}_{n}(X, A) & =h_{n}(X / A, *), \\
\hat{h}_{n}(f) & =h_{n}(\bar{f}) .
\end{aligned}
$$

For any pair $(X, A) \in \mathbf{C M}^{2}$ we have a commutative diagram

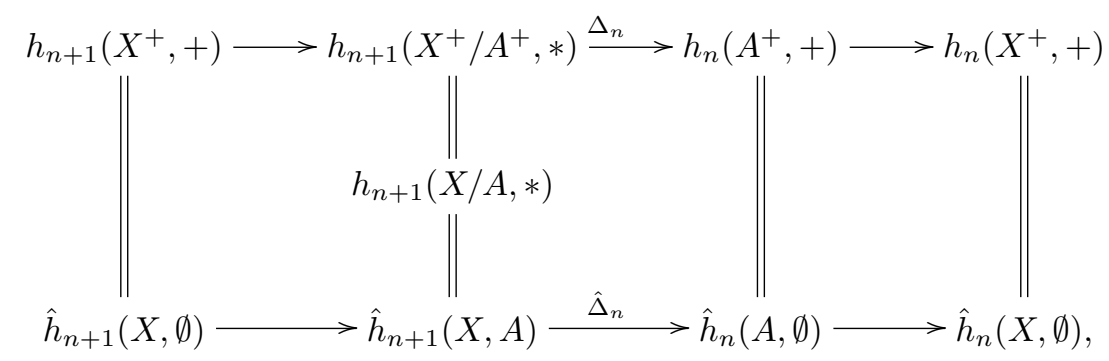

where the top row is the long exact sequence of the pointed pair $\left(X^{+}, A^{+},+\right)$and $\hat{\Delta}_{n}$ is defined to make the middle square commutative. 
It is then clear that $\left(\hat{h}_{n}, \hat{\Delta}_{n}\right)$ is an unreduced homology theory on $\mathbf{C M}^{\mathbf{2}}$ which by construction satisfies the strong excision axiom (since $(X / A) /\{*\}=X / A)$.

It is easy to see that the unreduced homology theories $\left(\hat{\bar{H}}_{n}, \hat{\bar{\partial}}_{n}\right)$ and $\left(H_{n}, \partial_{n}\right)$ are naturally isomorphic: Observe that

$$
\hat{\bar{H}}_{n}(X, A)=H_{n}(X / A,\{*\})
$$

and verify that the strong excision isomorphism $p_{*}: H_{n} \rightarrow \hat{\bar{H}}_{n}$ is an isomorphism of unreduced homology theories.

It is also easy to see that the reduced pointed Q-homology theories $\left(\overline{\hat{h}}_{n}, \overline{\hat{\Delta}}_{n}\right)$ and $\left(h_{n}, \Delta_{n}\right)$ are naturally isomorphic: Observe that

$$
\overline{\hat{h}}_{n}\left(X, x_{0}\right)=h_{n}\left(X /\left\{x_{0}\right\}, *\right)
$$

and verify that the pointed homeomorphisms $q^{\left(X, x_{0}\right)}:\left(X, x_{0}\right) \rightarrow\left(X /\left\{x_{0}\right\}, *\right)$ induce an isomorphism $q_{*}: h_{n} \rightarrow \overline{\hat{h}}_{n}$ of reduced pointed Q-homology theories.

Remark 6.4. It follows from Theorems 6.2 and 6.3 that strong shape invariant reduced pointed homology theories on $\mathbf{C M}_{\mathbf{0}}$ are equivalent to strong shape invariant reduced unpointed homology theories on $\mathbf{C M}_{\mathbf{n e}}$. Using the fact that $X$ is a strong deformation retract of $X \cup C\left\{x_{0}\right\}$, one easily verifies the following: Given a reduced unpointed homology theory $\left(h_{n}, \sigma_{n}\right)$ on $\mathbf{C M}_{\mathbf{n e}}$, an equivalent reduced pointed homology theory $\left(\tilde{h}_{n}, \tilde{\sigma}_{n}\right)$ on $\mathbf{C M}_{\mathbf{0}}$ is obtained by

$$
\tilde{h}_{n}\left(X, x_{0}\right)=h_{n}(X), \tilde{h}_{n}(f)=h_{n}(f),
$$

i.e., by forgetting the basepoints.

Corollary 6.5. Let $\left(h_{n}, \sigma_{n}\right)$ be a quotient exact reduced pointed homology system on $\mathbf{C M}_{\mathbf{0}}$. If two pointed maps $f, g:\left(X, x_{0}\right) \rightarrow\left(Y, y_{0}\right)$ between pointed compacta induce the same unpointed strong shape morphism, then they induce the same homomorphism $f_{*}=g_{*}: h_{n}\left(X, x_{0}\right) \rightarrow h_{n}\left(Y, y_{0}\right)$. This applies, in particular, if $f, g$ are freely homotopic.

\section{Pointed cylinder base embeddings}

It is well-known that in the absolute case the strong shape functor $S: \mathbf{C M} \rightarrow \mathbf{S S h}$ localizes CM at the class of strong shape equivalences see, e.g., [16, Section 10.7]). In $[\mathbf{1 8}]$ we have shown that this generalizes to compact metric pairs. In this section we treat the pointed case.

The pointed strong shape category $\mathbf{S S h}_{\mathbf{0}}$ can be regarded as a full subcategory of the strong shape category of compact metric pairs $\mathbf{S S h}^{\mathbf{2}}$. This follows from the "standard" constructions of $\mathbf{S} \mathbf{S h}^{\mathbf{2}}$ and $\mathbf{S} \mathbf{S h}_{\mathbf{0}}$ as presented in [16]. The construction of $\mathbf{S S h}^{2}$ is based on resolutions of pairs, the construction of $\mathbf{S S h}_{\mathbf{0}}$ on pointed resolutions which occur as a special case of resolutions of pairs. See also [9] for an explicit reference.

We can therefore apply the results of $[\mathbf{1 8}]$. We recall that a cylinder base embedding of pairs is an embedding of compact metric pairs $i:\left(B, B_{0}\right) \rightarrow\left(C, C_{0}\right)$ such 
that $C_{0} \backslash i\left(B_{0}\right) \subset C \backslash i(B)$ and $\left(C \backslash i(B), C_{0} \backslash i\left(B_{0}\right)\right) \approx\left(Z, Z_{0}\right) \times(0,1]$ for some compact metric pair $\left(Z, Z_{0}\right)$.

Definition 7.1. A cylinder base embedding for pointed compacta is a cylinder base embedding of pairs $i:\left(B, B_{0}\right) \rightarrow\left(C, C_{0}\right)$ such that $B_{0}=\left\{b_{0}\right\}$ for some point $b_{0} \in B$ and $\left(C \backslash i(B), C_{0} \backslash i\left(B_{0}\right)\right) \approx\left(Z, z_{0}\right) \times(0,1]$ for some pointed compactum $\left(Z, z_{0}\right)$.

By [18, Theorem A.1] each cylinder base embedding for pointed compacta is a strong shape equivalence of pairs of compacta. Observe that $C_{0} \backslash\left\{i\left(b_{0}\right)\right\} \approx$ $\left\{z_{0}\right\} \times(0,1]$. Thus $C_{0}$ is a homeomorphic copy of the unit interval $I$. Note also that $i(B) \cap C_{0}=\left\{i\left(b_{0}\right)\right\}$.

Definition 7.2. A collapse is a map $p:\left(C, C_{0}\right) \rightarrow\left(D, d_{0}\right)$ from a compact metric pair $\left(C, C_{0}\right)$ to a pointed metric space $\left(D, d_{0}\right)$ such that $p$ maps $C \backslash C_{0}$ homeomorphically onto $D \backslash\left\{d_{0}\right\}$.

For each compact metric pair $\left(C, C_{0}\right)$, the quotient map $q:\left(C, C_{0}\right) \rightarrow\left(C / C_{0},\left[C_{0}\right]\right)$ is a collapse. For any collapse $p:\left(C, C_{0}\right) \rightarrow\left(D, d_{0}\right)$ there is a unique homeomorphism $\kappa:\left(C / C_{0},\left[C_{0}\right]\right) \rightarrow\left(D, d_{0}\right)$ such that $\kappa \circ q=p$.

Definition 7.3. A map of pointed compacta $j:\left(B, b_{0}\right) \rightarrow\left(D, d_{0}\right)$ is called a pointed cylinder base embedding if it can be decomposed as $j=p \circ i$, where $i:\left(B, b_{0}\right) \rightarrow$ $\left(C, C_{0}\right)$ is a cylinder base embedding for pointed compacta and $p:\left(C, C_{0}\right) \rightarrow\left(D, d_{0}\right)$ is a collapse.

Note that $j$ is in fact an embedding because $i(B) \cap C_{0}=\left\{i\left(b_{0}\right)\right\}$. Also observe that the collapse $p$ occurring in the definition of a pointed cylinder base embedding is a strong shape equivalence of pairs since $C_{0}$ is a homeomorphic copy of the unit interval (see $[\mathbf{9}])$.

Proposition 7.4. Let $j:\left(B, b_{0}\right) \rightarrow\left(D, d_{0}\right)$ be a pointed cylinder base embedding. Then the quotient space $(D / j(B),[j(B)])$ obtained from $\left(D, d_{0}\right)$ by collapsing its base $j(B)$ to a point is pointed contractible.

Proof. Let $i:\left(B, b_{0}\right) \rightarrow\left(C, C_{0}\right)$ be as in Definition 7.3. Let $\left(Z, z_{0}\right)$ be a pointed compactum such that $\left(C \backslash i(B), C_{0} \backslash i\left(b_{0}\right)\right) \approx\left(Z, z_{0}\right) \times(0,1]$. Then $(D / j(B),[j(B)]) \approx$ $C\left(Z, z_{0}\right)=$ reduced cone of $\left(Z, z_{0}\right)$.

Proposition 7.5. Each pointed cylinder base embedding is a pointed strong shape equivalence of pointed compacta.

Proof. By definition, each pointed cylinder base embedding is the composition of two strong shape equivalences of pairs of compacta and therefore is itself a strong shape equivalence of pairs of compacta. Since $\mathbf{S S h}$ is a full subcategory of $\mathbf{S S h}^{\mathbf{2}}$, we are done.

Proposition 7.6. Let $\Phi:\left(X, x_{0}\right) \rightarrow\left(Y, y_{0}\right)$ be a pointed strong shape morphism between pointed compacta. Then there exist a pointed cylinder base embedding $j:\left(Y, y_{0}\right) \rightarrow\left(D, d_{0}\right)$ and a pointed map $f:\left(X, x_{0}\right) \rightarrow\left(D, d_{0}\right)$ such that $\Phi=S(j)^{-1} \circ$ $S(f)$. 
Proof. By [18], $\Phi$ has a mapping cylinder in the category $\mathbf{S S h}^{\mathbf{2}}$. This means that there exist a cylinder base embedding for pointed compacta $i:\left(Y, y_{0}\right) \rightarrow\left(C, C_{0}\right)$ and a map $g:\left(X, x_{0}\right) \rightarrow\left(C, C_{0}\right)$ such that $\Phi=S(i)^{-1} \circ S(g)$ in $\mathbf{S S h}^{2}$. Let $p:\left(C, C_{0}\right) \rightarrow\left(D, d_{0}\right)$ be a collapse. Then $j=p \circ i$ is a pointed cylinder base embedding. Letting $f=p \circ g$, we see that $\Phi=S(j)^{-1} \circ S(f)$ in $\mathbf{S S h}_{\mathbf{0}}$.

Let $\Sigma_{C B E_{0}}$ be the class of pointed cylinder base embeddings of pointed compacta.

Theorem 7.7. $S: \mathbf{C M}_{\mathbf{0}} \rightarrow \mathbf{S S h}_{\mathbf{0}}$ localizes $\mathbf{C M}_{\mathbf{0}}$ at $\Sigma_{C B E_{0}}$. A fortiori $S$ localizes $\mathbf{C M}_{\mathbf{0}}$ at pointed strong shape equivalences of pointed compacta.

To prepare the proof we recall the concept of a calculus of left fractions. Let $\mathbf{C}$ be a category and $\Sigma$ a set of morphisms in C. $\Sigma$ admits a calculus of left fractions if the following conditions are satisfied:

(LF0) For each object $X$ in $\mathbf{C}$ there is a set $\Sigma_{X} \subset \Sigma$ such that for each morphism $s: X \rightarrow Y$ of $\Sigma$ there exists an isomorphism $u: Y \rightarrow Y^{\prime}$ in $\mathbf{C}$ such that $u \circ s \in \Sigma_{X}$.

(LF1) $\Sigma$ contains all identity morphisms in $\mathbf{C}$.

(LF2) If $s: X \rightarrow Y$ and $t: Y \rightarrow Z$ are in $\Sigma$, then also $t \circ s$ is in $\Sigma$.

(LF3) Each pair of morphisms $X^{\prime} \stackrel{s}{\leftarrow} X \stackrel{u}{\rightarrow} Y$ with $s$ in $\Sigma$ can be completed to a commutative square

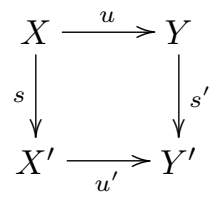

such that $s^{\prime}$ is in $\Sigma$.

(LF4) Let $u, v: X \rightarrow Y$ be morphisms in C. If there exists a morphism $s: X^{\prime} \rightarrow X$ in $\Sigma$ with $u \circ s=v \circ s$, then there exists a morphism $t: Y \rightarrow Y^{\prime}$ in $\Sigma$ with $t \circ u=t \circ v$.

If $\Sigma$ admits a calculus of left fractions, then we can construct the category of left fractions $\mathbf{C} / \Sigma$. It has the same objects as $\mathbf{C}$ and the morphisms from $X$ to $Y$ are equivalence classes of pairs of morphisms $(f: X \rightarrow Z, s: Y \rightarrow Z$ ) in $\mathbf{C}$ with $s \in \Sigma$ (for details see [8]). The equivalence class of $(f, s)$ is denoted by $f \backslash s$. A canonical quotient functor $Q: \mathbf{C} \rightarrow \mathbf{C} / \Sigma$ is defined by $Q(X)=X$ for the objects and $Q(f)=f \backslash$ id for the morphisms. This functor $Q$ localizes $\mathbf{C}$ at the class $\Sigma$.

We need a slight modification of the concept of a calculus of left fractions.

Definition 7.8. Let $\mathbf{C}$ be a category and $\Sigma$ a set of morphisms in $\mathbf{C}$. We say that $\Sigma$ admits a weak calculus of left fractions if conditions (LF0), (LF3) and (LF4) are satisfied.

Definition 7.9. Let $\Sigma$ admit a weak calculus of fractions. The closure of $\Sigma$ is defined to be the class $\bar{\Sigma}$ of all identities in $\mathbf{C}$ and all finite compositions $s_{n} \circ \cdots \circ s_{1}$ of elements $s_{1}, \ldots, s_{n} \in \Sigma$ (with arbitrary length $n$ ). 
Proposition 7.10. Let $\Sigma$ admit a weak calculus of fractions. Then the closure $\bar{\Sigma}$ admits a calculus of left fractions. The quotient functor $Q: \mathbf{C} \rightarrow \mathbf{C} \backslash \bar{\Sigma}$ localizes $\mathbf{C}$ at $\Sigma$.

Proof. This is a straightforward exercise.

Let $\mathbf{H C M}_{\mathbf{0}}$ be the homotopy category of $\mathbf{C M}_{\mathbf{0}}$ and let $H \Sigma_{C B E_{0}}$ be the set of homotopy classes of maps in $\Sigma_{C B E_{0}}$.

Theorem 7.11. $H \Sigma_{C B E_{0}}$ admits a weak calculus of left fractions in $\mathbf{H C M}_{\mathbf{0}}$.

Proof of (LF0). (LF0) is obvious since each pointed compactum has a homeomorphic copy in the Hilbert cube.

Proof of (LF3). Let $\left(X^{\prime}, x_{0}^{\prime}\right) \stackrel{j}{\leftarrow}\left(X, x_{0}\right) \stackrel{u}{\rightarrow}\left(Y, y_{0}\right)$ be a pair of morphisms in $\mathbf{C M}_{\mathbf{0}}$ with $j \in \Sigma_{C B E_{0}}$. We have $j=p \circ i$, where $i:\left(X, x_{0}\right) \rightarrow\left(C, C_{0}\right)$ is a cylinder base embedding for pointed compacta, and $p:\left(C, C_{0}\right) \rightarrow\left(X^{\prime}, x_{0}^{\prime}\right)$ is the collapse associated to $i$. Consider the pushout diagram

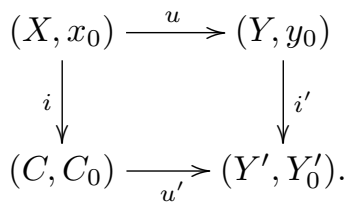

It is easy to see that $i^{\prime}$ is a a cylinder base embedding for pointed compacta and that $u^{\prime}$ maps $C_{0}$ homeomorphically onto $Y_{0}^{\prime}$.

Let $p^{\prime}:\left(Y^{\prime}, Y_{0}^{\prime}\right) \rightarrow\left(Y^{\prime \prime}, y_{0}^{\prime \prime}\right)$ be the collapse associated to $i^{\prime}$. Then $u^{\prime}$ induces a map $u^{\prime \prime}:\left(X^{\prime}, x_{0}^{\prime}\right) \rightarrow\left(Y^{\prime \prime}, y_{0}^{\prime \prime}\right)$ such that the diagram

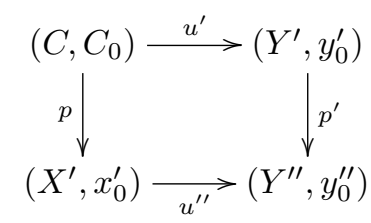

commutes. Since $j^{\prime}=p^{\prime} \circ i^{\prime}:\left(Y, y_{0}\right) \rightarrow\left(Y^{\prime \prime}, y_{0}^{\prime \prime}\right)$ is a pointed cylinder base embedding, we are done.

Proof of (LF4). Let $f_{0}$ and $f_{1}:\left(X, x_{0}\right) \rightarrow\left(Y, y_{0}\right)$ be two pointed maps and let $j:\left(X^{\prime}, x_{0}^{\prime}\right) \rightarrow\left(X, x_{0}\right)$ be a pointed cylinder base embedding such that $f_{0} \circ j \simeq f_{1} \circ j$ $(\simeq$ denotes basepoint preserving homotopy). By definition, there exist a cylinder base embedding for pointed compacta $i:\left(X^{\prime}, x_{0}^{\prime}\right) \rightarrow\left(C, C_{0}\right)$ and a collapse $p:\left(C, C_{0}\right) \rightarrow$ $\left(X, x_{0}\right)$ such that $p \circ i=j$.

There is a homotopy $H:\left(j\left(X^{\prime}\right), x_{0}\right) \times I \rightarrow\left(Y, y_{0}\right)$ such that $H_{0}=\left.f_{0}\right|_{j\left(X^{\prime}\right)}$ and $H_{1}=\left.f_{1}\right|_{j\left(X^{\prime}\right)}$. We can extend $H$ to a map

$$
H^{\prime}:\left(X \times\{0,1\} \cup j\left(X^{\prime}\right) \times I, x_{0} \times I\right) \rightarrow\left(Y, y_{0}\right)
$$

such that $H_{0}^{\prime}=f_{0}$ and $H_{1}^{\prime}=f_{1}$. 
Let

$$
\iota:\left(X \times\{0,1\} \cup j\left(X^{\prime}\right) \times I, x_{0} \times I\right) \rightarrow\left(X, x_{0}\right) \times I
$$

denote inclusion and let

$$
\left(D, d_{0}\right)=\left(\left(X \times\{0,1\} \cup j\left(X^{\prime}\right) \times I\right) / x_{0} \times I,\left[x_{0} \times I\right]\right)
$$

denote the pointed quotient space obtained by contracting $x_{0} \times I$ to a point. We have quotient maps

$$
q:\left(X \times\{0,1\} \cup j\left(X^{\prime}\right) \times I, x_{0} \times I\right) \rightarrow\left(D, d_{0}\right)
$$

and

$$
q^{\prime}:\left(X, x_{0}\right) \times I \rightarrow\left(X \times I / x_{0} \times I,\left[x_{0} \times I\right]\right) .
$$

$H^{\prime}$ induces a pointed map

$$
H^{\prime \prime}:\left(D, d_{0}\right) \rightarrow\left(Y, y_{0}\right)
$$

such that $H^{\prime \prime} \circ q=H^{\prime}$ and $\iota$ induces an embedding

$$
\iota^{\prime}:\left(D, d_{0}\right) \rightarrow\left(X \times I / x_{0} \times I,\left[x_{0} \times I\right]\right)
$$

of pointed compacta such that $\iota^{\prime} \circ q=q^{\prime} \circ \iota$. We shall show that $\iota^{\prime}$ is a pointed cylinder embedding. Let $R=C \times\{0,1\} \cup i\left(X^{\prime}\right) \times I$ and $R_{0}=C_{0} \times\{0,1\} \cup i\left(x_{0}^{\prime}\right) \times I$. The map $p \times \operatorname{id}_{I}:\left(C, C_{0}\right) \times I \rightarrow\left(X, x_{0}\right) \times I$ restricts to a map

$$
\bar{p}:\left(R, R_{0}\right) \rightarrow\left(X \times\{0,1\} \cup j\left(X^{\prime}\right) \times I, x_{0} \times I\right) .
$$

Let

$$
r:\left(R, R_{0}\right) \rightarrow\left(R / R_{0},\left[R_{0}\right]\right)
$$

denote the quotient map. It is easy to see that $q \circ \bar{p}$ induces a map

$$
\hat{p}:\left(R / R_{0},\left[R_{0}\right]\right) \rightarrow\left(D, d_{0}\right)
$$

such that $\hat{p} \circ r=q \circ \bar{p}$. Since $p$ is a collapse, $\hat{p}$ is a homeomorphism.

We shall first show that the inclusion

$$
\iota^{\prime \prime}:\left(R, R_{0}\right) \rightarrow\left(C \times I, C_{0} \times I\right)
$$

is a cylinder base embedding of compact metric pairs.

Since $i$ is a cylinder base embedding for pointed compacta, there exist a pointed compactum $\left(Z, z_{0}\right)$ and a homeomorphism

$$
h:\left(Z, z_{0}\right) \times(0,1] \rightarrow\left(C \backslash i\left(X^{\prime}\right), C_{0} \backslash i\left(x_{0}^{\prime}\right)\right) .
$$

Let

$$
h_{0}:\left\{z_{0}\right\} \times(0,1] \rightarrow C_{0} \backslash i\left(x_{0}^{\prime}\right)
$$

denote the restriction of $h$. Clearly $h_{0}$ extends to a homeomorphism

$$
k_{0}:\left\{z_{0}\right\} \times I \rightarrow C_{0} .
$$

Define a homeomorphism $h^{\prime}$ by

$$
\begin{aligned}
h^{\prime} & =h \times \operatorname{id}_{(0,1)}:\left(Z, z_{0}\right) \times(0,1] \times(0,1) \rightarrow\left(C \backslash i\left(X^{\prime}\right), C_{0} \backslash i\left(x_{0}^{\prime}\right)\right) \times(0,1) \\
& =\left(C \times I \backslash R, C_{0} \times I \backslash R_{0}\right)
\end{aligned}
$$


and let

$$
h_{0}^{\prime}:\left\{z_{0}\right\} \times(0,1] \times(0,1) \rightarrow C_{0} \times I \backslash R_{0}
$$

denote the restriction of $h^{\prime}$. By construction, the homeomorphism

$$
k_{0}^{\prime}=k_{0} \times \operatorname{id}_{I}:\left\{z_{0}\right\} \times I \times I \rightarrow C_{0} \times I
$$

is an extension of $h_{0}^{\prime}$.

Choose a homeomorphism $\lambda: I \times I \rightarrow I \times I$ such that $\lambda(I \times(0,1])=(0,1] \times(0,1)$. We obtain a homeomorphism

$$
h^{\prime \prime}=h^{\prime} \circ\left(\operatorname{id}_{Z} \times \lambda\right):\left(Z, z_{0}\right) \times I \times(0,1] \rightarrow\left(C \times I \backslash R, C_{0} \times I \backslash R_{0}\right)
$$

which shows that $\iota^{\prime \prime}$ is a cylinder base embedding of compact metric pairs.

Next let

$$
h_{0}^{\prime \prime}:\left\{z_{0}\right\} \times I \times(0,1] \rightarrow C_{0} \times I \backslash R_{0}
$$

denote the restriction of $h^{\prime \prime}$. Again by construction, the homeomorphism

$$
k_{0}^{\prime \prime}=k_{0}^{\prime} \circ\left(\operatorname{id}_{z_{0}} \times \lambda\right):\left\{z_{0}\right\} \times I \times I \rightarrow C_{0} \times I
$$

is an extension of $h_{0}^{\prime \prime}$.

The fibres $F_{t}=k_{0}^{\prime \prime}\left(\left\{z_{0}\right\} \times I \times\{t\}\right), t \in I$, are homeomorphic copies of the unit interval $I$. We have $F_{0}=R_{0}$ and $F_{t} \cap R=\emptyset$ for $t \in(0,1]$. Define an equivalence relation $\sim$ on $C \times I$ by

$$
\xi_{1} \sim \xi_{2} \text { if and only if } \xi_{1}=\xi_{2} \text { or there exists } t \in I \text { such that } \xi_{1}, \xi_{2} \in F_{t} .
$$

$\sim$ restricts to an equivalence relation on $C_{0}$. Define

$$
\left(E, E_{0}\right)=\left(C \times I / \sim, C_{0} \times I / \sim\right) .
$$

$\left(E, E_{0}\right)$ is a compact metric pair such that $E_{0}$ is a homeomorphic copy of $I$. Let $\pi:\left(C \times I, C_{0} \times I\right) \rightarrow\left(E, E_{0}\right)$ be the quotient map. It contracts each fibre $F_{t} \subset C_{0}$ to a point and compresses the square $C_{0} \times I$ to $E_{0}$. Since $\pi\left(R_{0}\right)=\left[F_{0}\right] \in\left[E_{0}\right]$, the restriction of $\pi$ to $\left(R, R_{0}\right)$ induces a map

$$
\pi^{\prime}:\left(R / R_{0},\left[R_{0}\right]\right) \rightarrow\left(E, E_{0}\right)
$$

such that $\pi \circ \iota^{\prime \prime}=\pi^{\prime} \circ r$. Since $F_{t} \cap R=\emptyset$ for $t \in(0,1], \pi^{\prime}$ is an embedding.

By construction, $h^{\prime \prime}:\left(Z, z_{0}\right) \times I \times(0,1] \rightarrow\left(C \times I \backslash R, C_{0} \times I \backslash R_{0}\right)$ maps the fibres $F_{t}^{\prime}=\left\{z_{0}\right\} \times I \times\{t\}$ homeomorphically onto

$$
h^{\prime \prime}\left(F_{t}^{\prime}\right)=h_{0}^{\prime \prime}\left(F_{t}^{\prime}\right)=k_{0}^{\prime \prime}\left(F_{t}^{\prime}\right)=F_{t}, t \in(0,1] .
$$

Hence $h^{\prime \prime}$ induces a homeomorphism

$$
h^{\prime \prime \prime}:\left(Z /\left\{z_{0}\right\} \times I,\left[\left\{z_{0}\right\} \times I\right]\right) \times(0,1] \rightarrow\left(E \backslash \pi^{\prime}\left(R / R_{0}\right), E_{0} \backslash \pi^{\prime}\left(\left[R_{0}\right]\right)\right) .
$$

This means that $\pi^{\prime}$ is a cylinder base embedding for pointed compacta.

Consider the map

$$
q^{\prime} \circ\left(p \times \operatorname{id}_{I}\right):\left(C \times I, C_{0} \times I\right) \rightarrow\left(X \times I / x_{0} \times I,\left[x_{0} \times I\right]\right) .
$$

It is a collapse which contracts the square $C_{0} \times I$ to the point $\left[x_{0} \times I\right]$. Since it maps points which are equivalent with respect to $\sim$ to the same point in $X \times I / x_{0} \times I$, it 
induces a map

$$
s:\left(E, E_{0}\right) \rightarrow\left(X \times I / x_{0} \times I,\left[x_{0} \times I\right]\right) .
$$

It is clear by construction that $s$ is a collapse. Therefore

$$
J=s \circ \pi^{\prime}:\left(R / R_{0},\left[R_{0}\right]\right) \rightarrow\left(X \times I / x_{0} \times I,\left[x_{0} \times I\right]\right)
$$

is a pointed cylinder base embedding. It is easy to verify that $J=\iota^{\prime} \circ \hat{p}$. Since $\hat{p}$ is a homeomorphism, we conclude that $\iota^{\prime}$ is a pointed cylinder base embedding as we claimed above.

As in the proof of (LF3) there exists a commutative diagram

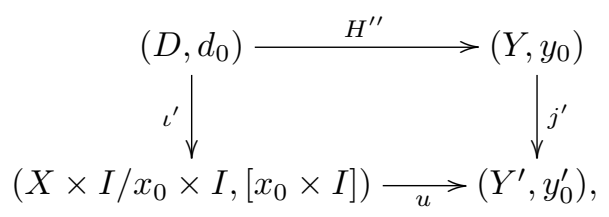

where $j^{\prime}$ is a pointed cylinder base embedding. We therefore obtain a commutative diagram

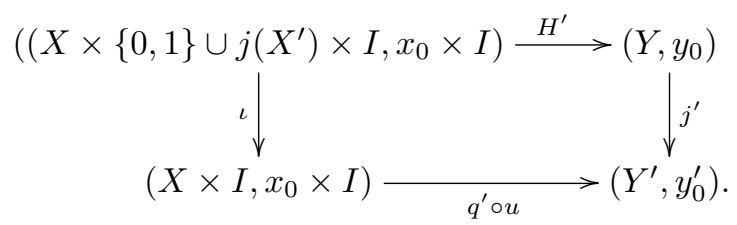

The pointed homotopy $G=q^{\prime} \circ u$ satisfies $G_{0}=j^{\prime} \circ H_{0}^{\prime}=\iota \circ f_{0}$ and $G_{1}=j^{\prime} \circ f_{1}$ which proves (LF4).

We now prove Theorem 7.7 .

Let us first consider any functor $\Theta: \mathbf{C M}_{\mathbf{0}} \rightarrow \mathbf{K}$ taking $\Sigma_{C B E_{0}}$ to isomorphisms in the category $\mathbf{K}$. Then $\Theta$ is homotopy invariant, i.e., factorizes as $\Theta=H \Theta \circ H$ with $H \Theta: \mathbf{H C M}_{\mathbf{0}} \rightarrow \mathbf{K}$. This comes from the fact that the inclusions $i_{k}:\left(X, x_{0}\right) \rightarrow$ $\left(X, x_{0}\right) \times I /\left\{x_{0}\right\} \times I, i_{k}(x)=[x, k], k=0,1$ are pointed cylinder base embeddings and therefore induce isomorphisms $\Theta\left(i_{k}\right)$. Hence $\Theta\left(i_{0}\right)=\Theta\left(i_{1}\right)$ since $\Theta(p)$, where $p:\left(X, x_{0}\right) \times I /\left\{x_{0}\right\} \times I \rightarrow\left(X, x_{0}\right)$ denotes the projection map, is a common right inverse for both. This implies that homotopic maps have the same image under $\Theta$.

It is therefore sufficient to show that the functor $H S: \mathbf{H C M}_{\mathbf{0}} \rightarrow \mathbf{S S h}_{\mathbf{0}}$ localizes $\mathbf{H C M}_{\mathbf{0}}$ at $H \Sigma_{C B E_{0}}$.

Consider the closure $\overline{H \Sigma_{C B E_{0}}}$ of $H \Sigma_{C B E_{0}}$. Let $\mathbf{H C M}_{\mathbf{0}} / \overline{H \Sigma_{C B E_{0}}}$ be the category of left fractions and let $\Lambda: \mathbf{H C M}_{\mathbf{0}} \rightarrow \mathbf{H C M}_{\mathbf{0}} / \overline{H \Sigma_{C B E_{0}}}$ be the quotient functor which localizes $\mathbf{H C M}_{\mathbf{0}}$ at $H \Sigma_{C B E_{0}}$ (cf. Proposition 7.10).

By Proposition 7.5, $H S$ takes $H \Sigma_{C B E_{0}}$ to isomorphisms. Hence $H S$ also takes the closure $\overline{H \Sigma_{C B E_{0}}}$ to isomorphisms. Therefore there exists a unique functor

$$
\Omega: \mathbf{H C M}_{\mathbf{0}} / \overline{H \Sigma_{C B E_{0}}} \rightarrow \mathbf{S S h}_{\mathbf{0}}
$$

such that $H S=\Omega \circ \Lambda$. On the morphisms, $\Omega([f] \backslash[s])=S([s])^{-1} \circ S([f])$. We shall show that $\Omega$ is a category isomorphism; this will prove Theorem 7.7 . 
By Proposition 7.6, $\Omega$ is a full functor. We shall show that $\Omega$ is faithful. Using the fact that $\overline{H \Sigma_{C B E_{0}}}$ admits a calculus of left fractions, it suffices to show the following (see, e.g., [4, Lemma 1.19]):

For any pointed homotopy classes $\left[f_{0}\right],\left[f_{1}\right]:\left(X, x_{0}\right) \rightarrow\left(Y, y_{0}\right)$ such that $H S\left(\left[f_{0}\right]\right)=$ $H S\left(\left[f_{1}\right]\right)$, there exists $[s] \in \overline{H \Sigma_{C B E_{0}}}$ such that $[s] \circ\left[f_{0}\right]=[s] \circ\left[f_{1}\right]$.

We shall show that we can even find an $[s] \in H \Sigma_{C B E_{0}}$ with this property. This will be done using mapping cylinders of approaching maps (see [17]). We may assume that $X$ and $Y$ are contained in the Hilbert cube $Q$. Then we can identify pointed strong shape morphisms $\left(X, x_{0}\right) \rightarrow\left(Y, y_{0}\right)$ with approaching homotopy classes of approaching maps $\left(Q, X, x_{0}\right) \Rightarrow\left(Q, Y, y_{0}\right)$.

Choose approaching extensions

$$
\varphi_{k}:\left(Q, X, x_{0}\right) \Rightarrow\left(Q, Y, y_{0}\right) \quad \text { of } \quad f_{k}:\left(X, x_{0}\right) \rightarrow\left(Y, y_{0}\right) .
$$

By assumption, there exists an approaching homotopy $\phi:\left(Q, X, x_{0}\right) \times I \Rightarrow\left(Q, Y, y_{0}\right)$ connecting $\varphi_{0}$ and $\varphi_{1}$. We identify the mapping cylinders $C^{2}\left(\varphi_{k}\right)=C^{2}\left(f_{k}\right)$ with the left and right face of the mapping cylinder $C^{2}(\phi)$ (as sets, $C^{2}\left(g_{k}\right)=\left(X, x_{0}\right)$ $\left.\times\{k\} \times(0,1]+\left(Y, y_{0}\right) \subset\left(X, x_{0}\right) \times I \times(0,1]+\left(Y, y_{0}\right)=C^{2}(\phi)\right)$. We choose a homeomorphism

$$
h:\left(X, x_{0}\right) \times I \times(0,1] \rightarrow C^{2}(\phi) \backslash\left(Y, y_{0}\right) .
$$

Let $\alpha_{k}: C^{2}\left(f_{k}\right) \rightarrow C^{2}(\phi)$ be the left and right face embeddings, let $i_{\left(X, x_{0}\right)}^{(k)}:\left(X, x_{0}\right) \rightarrow$ $C^{2}\left(f_{k}\right)$ be the top embeddings (identifying $\left(X, x_{0}\right)$ with $\left.h\left(\left(X, x_{0}\right) \times\{k\} \times\{1\}\right)\right)$ and let $j_{\left(Y, y_{0}\right)}^{(k)}:\left(Y, y_{0}\right) \rightarrow C^{2}\left(f_{k}\right)$ as well as $j_{\left(Y, y_{0}\right)}:\left(Y, y_{0}\right) \rightarrow C^{2}(\phi)$ be the base embeddings. We then have

$$
\begin{aligned}
j_{\left(Y, y_{0}\right)} \circ f_{0}=\alpha_{0} \circ j_{\left(Y, y_{0}\right)}^{(0)} \circ f_{0} & \simeq \alpha_{0} \circ i_{\left(X, x_{0}\right)}^{(0)} \\
& \simeq \alpha_{1} \circ i_{\left(X, x_{0}\right)}^{(1)} \\
& \simeq \alpha_{1} \circ j_{\left(Y, y_{0}\right)}^{(1)} \circ f_{1}=j_{\left(Y, y_{0}\right)} \circ f_{1} .
\end{aligned}
$$

Here, $\simeq$ denotes homotopy of pairs.

We have $C^{2}(\phi)=\left(C(\phi), C\left(\phi_{0}\right)\right)$, where $\phi_{0}:\left(Q, x_{0}\right) \times I \Rightarrow\left(Q, y_{0}\right)$ is the restriction of $\phi$. Let

$$
q: C^{2}(\phi) \rightarrow\left(C(\phi) / C\left(\phi_{0}\right),\left[C\left(\phi_{0}\right)\right]\right)
$$

be the quotient map. Defining $s=q \circ j_{\left(Y, y_{0}\right)}$, we see that $s \circ f_{0}$ and $s \circ f_{1}$ are pointed homotopic. We shall show that $s$ is a pointed cylinder base embedding.

Define an equivalence relation $\sim$ on $C(\phi)$ by

$$
\begin{array}{r}
\xi_{1} \sim \xi_{2} \text { if and only if } \xi_{1}=\xi_{2} \text { or there exists } t \in(0,1] \\
\text { such that } \xi_{1}, \xi_{2} \in h\left(x_{0} \times I \times\{t\}\right) .
\end{array}
$$

$\sim$ restricts to an equivalence relation on $C\left(\phi_{0}\right)$. Let

$$
r: C^{2}(\phi) \rightarrow\left(C(\phi) / \sim, C\left(\phi_{0}\right) / \sim\right)
$$

denote the quotient map. Then $i=r \circ j_{\left(Y, y_{0}\right)}$ is a cylinder base embedding for pointed 
compacta since $h$ induces a homeomorphism

$$
\left((C(\phi) / \sim) \backslash i(Y),\left(C\left(\phi_{0}\right) / \sim\right) \backslash i\left(y_{0}\right)\right) \approx\left(X \times I / x_{0} \times I,\left[x_{0} \times I\right]\right) \times(0,1] .
$$

It is clear that $q$ maps points which are equivalent with respect to $\sim$ to the same point in $X \times I / x_{0} \times I$. Therefore $q$ induces a map

$$
p:\left((C(\phi) / \sim) \backslash i(Y),\left(C\left(\phi_{0}\right) / \sim\right) \backslash i\left(y_{0}\right)\right) \rightarrow\left(C(\phi) / C\left(\phi_{0}\right),\left[C\left(\phi_{0}\right)\right]\right)
$$

such that $p \circ r=q$. This map obviously is a collapse.

We have now shown that $s=q \circ j_{\left(Y, y_{0}\right)}=p \circ r \circ j_{\left(Y, y_{0}\right)}=p \circ i$ is a pointed cylinder base embedding which completes the proof. The a fortiori part of the theorem holds because pointed strong shape equivalences are precisely the morphisms which are taken to isomorphisms by $S$.

\section{References}

[1] F.W. Bauer, Under what conditions are shape homology $\underline{E}_{*}$ and Steenrod homology ${ }^{s} \underline{E}_{*}$ isomorphic?, in Shape theory and geometric topology, (Dubrovnik, 1981), Lecture Notes in Math. 870, Springer-Verlag, New York, 1981, 186-214.

[2] F.W. Bauer, Extensions of generalized homology theories, Pacific J. Math. 128 (1987), no. 1, 25-61.

[3] L.G. Brown, R.G. Douglas and P.A. Fillmore, Extensions of $C^{*}$-algebras and K-homology, Ann. of Math. 105 (1977), no. 2, 265-324. 265-324.

[4] F.W. Cathey, Strong shape theory, in Shape theory and geometric topology (S. Mardešić and J. Segal, eds.), Lecture Notes in Mathematics 870, SpringerVerlag, New York, 1981, 215-238.

[5] A. Dold and R. Thom, Quasifaserungen und unendliche symmetrische Produkte, Ann. of Math. 67 (1958), no. 2, 239-281.

[6] D.A. Edwards and H.M. Hastings, Cech and Steenrod homotopy theories with applications to geometric topology, Lecture Notes in Math. 542, SpringerVerlag, New York, 1976.

[7] S.C. Ferry, Remarks on Steenrod homology, in Novikov conjectures, index theorems and rigidity, Vol. 2 (Oberwolfach, 1993) (S.C. Ferry, A. Ranicki and J. Rosenberg, eds.), London Math. Soc. Lecture Note Series 227, Cambridge University Press, Cambridge, 1995, 148-166.

[8] P. Gabriel and M. Zisman, Calculus of fractions and homotopy theory, Ergebnisse der Mathematik und ihrer Grenzgebeite 35, Springer-Verlag, New York, 1967.

[9] B. Günther, Properties of normal embeddings concerning strong shape theory. II, Tsukuba J. Math. 16 (1992), no. 2, 429-438.

[10] A. Hatcher, Algebraic topology, Cambridge University Press, Cambridge, 2002.

[11] N. Higson and J. Roe, On the coarse Baum-Connes conjecture, in Novikov conjectures, index theorems and rigidity, Vol. 2 (S.C. Ferry, A. Ranicki and J. Rosenberg, eds.), London Math. Soc. Lecture Note Series 227, Cambridge University Press, Cambridge, 1995, 227-254. 
[12] S.-T. Hu, On axiomatic approach to homology theory without using the relative groups, Portugaliae Math. 19 (1960), 211-225.

[13] J. Kaminker and C. Schochet, $K$-theory and Steenrod homology: applications to the Brown-Douglas-Fillmore theory of operator algebras, Trans. Amer. Math. Soc. 227 (1977), 63-107.

[14] D.S. Kahn, J. Kaminker and C. Schochet, Generalized homology theories on compact metric spaces, Michigan Math. J. 24 (1977), 203-224.

[15] G.M. Kelly, Single-space axioms for homology theory, Proc. Cambridge Philos. Soc. 55 (1959), 10-22.

[16] S. Mardešić, Strong shape and homology, Springer-Verlag, New York, 2000.

[17] P. Mrozik, Mapping cylinders of approaching maps and strong shape, J. London Math. Soc. (2) 41 (1990), 159-174.

[18] P. Mrozik, Strong excision and strong shape invariance are equivalent on the category of compact metric pairs, Homology, Homotopy and Applications 10 (2008), no. 1, 345-351.

[19] R.M. Switzer, Algebraic topology -homotopy and homology, Springer-Verlag, New York, 1975.

Peter Mrozik pmrozik@gmx.de

Berliner Str. 11, 61194 Niddatal, Germany 